\title{
El Acuerdo de Paz de la Habana. Un estudio de percepción en la población del Tecnológico de Antioquia-IU
}

\author{
The Havana Peace Agreement. A study of perception in the population of the \\ Tecnológico de Antioquia-IU.
}

\author{
Alexandra Jima González \\ Miguel Paradela López \\ Hernán Darío Pineda Gómez \\ Sara Liliana Areiza Villa \\ César Augusto García Marín \\ Katherin Vanessa Loaiza Seguro \\ Juan Esteban Parra Tavera
}

Resumen: el presente artículo se enmarca en el proyecto de investigación que actualmente se realiza por parte del Semillero de Investigación "Globalización y Territorio" del Tecnológico de Antioquia-IU (TdeA). En él se aborda un estudio de percepción del plebiscito sobre el Acuerdo de Paz entre el gobierno colombiano y las Fuerzas Armadas Revolucionarias de Colombia-Ejército del Pueblo. Mediante un estudio transversal descriptivo, se realizaron encuestas a profesores, administrativos y estudiantes del TdeA. A los datos tabulados se les aplicó una regresión logística multinomial con el propósito de explorar las posibles relaciones entre los diferentes colectivos del TdeA con respecto a variables como: voto en el acuerdo, fuentes de información, conocimiento del contenido del acuerdo y percepción del mismo. Supone, en definitiva, un intento de estudiar cómo de informada se encuentra la población del TdeA con respecto al Acuerdo de Paz y cómo influye el nivel de conocimiento de los encuestados en su voto.

Palabras claves: acuerdo de paz, plebiscito, gobierno.

\begin{abstract}
The present research work emerged from the ongoing project implemented by the Semillero de Investigación "Globalización y Territorio" from Tecnológico de Antioquia-IU (TdeA). This project develops a perception study on the Peace Agreement referendum between the Colombian government and FARC-EP. Through a cross-sectional descriptive methodology, the study surveys a total of 618 members of the university's community (teachers, students and administrative members). After obtaining descriptive statistics, a multinomial logistic regression approach is applied in order to explore the relationships among the different groups from TdeA regarding variables such as: voting preference, sources of information, knowledge of the agreement's content and its perception. Hence, the study main aim is to analyze how well informed the TdeA community is regarding the content of the Peace Agreement, and how such level of information influenced in turn its voting preferences.
\end{abstract}

Keywords: peace agreement, plebiscite, government. 


\section{L'accord de paix de La Havane. Une étude de la perception dans la population du Tecnológico de Antioquia-IU}

Résumé: Cet article fait partie du projet de recherche actuellement en cours par la recherche Hotbed «Mondialisation et Territoire » Tecnológico de Antioquia-IU (TDEA). Il prévoit un référendum d'étude de la perception sur l'accord de paix entre le gouvernement colombien et les Forces armées révolutionnaires de l'Armée populaire de Colombie adressée. Grâce à une étude transversale descriptive, des enquêtes ont été menées auprès des professeurs, des administrateurs et des étudiants de la TdeA. Une des données sous forme de tableaux, il a appliqué une régression logistique multinomiale afin d'explorer les relations possibles entre les différents groupes de TDEA en ce qui concerne les variables telles que les sources d'information de l'accord de vote, la connaissance du contenu de l'accord et de la perception. Cela signifie, en bref, une tentative d'étudier la façon dont la population est informée TDEA en ce qui concerne l'Accord de paix et de la façon dont il influence le niveau des connaissances des personnes interrogées dans leur vote.

Most-clés: Accord de paix, plébiscite, gouvernement.

\section{O Acordo de Paz de Havana. Um estudo da percepção na população do Tecnológico de Antioquia-IU}

Resumo: Este artigo faz parte do projeto de pesquisa atualmente realizado pelo Seminário de Pesquisa "Globalização e Território" do Tecnológico de Antioquia-IU (TdeA). Trata-se de um estudo sobre a percepção do plebiscito sobre o Acordo de Paz entre o governo colombiano e as Forças Armadas Revolucionárias do Exército Popular da Colômbia. Por meio de um estudo transversal descritivo, foram realizadas pesquisas com professores, administradores e alunos do TdeA. Uma dados tabulados a aplicação de uma regressão logística multinomial, a fim de explorar as possíveis relações entre diferentes grupos da TDEA sobre variáveis como fontes de informação acordo de votos, o conhecimento do conteúdo do acordo e percepção. Trata-se, em suma, de uma tentativa de estudar como está informada a população do TdeA em relação ao Acordo de Paz e como o nível de conhecimento dos respondentes influencia seu voto.

Palavras-chave: acordo de paz, plebiscito, governo. 


\section{Introducción}

Durante la mayor parte del siglo XX Colombia estuvo inmersa en una serie de alzamientos armados, la mayoría de ellos en contra del Estado y el orden político establecido. Sin duda uno de los que más intensamente ha afectado al país ha sido el liderado por las Fuerzas Armadas Revolucionarias de Colombia (FARC-EP), tanto por su duración en el tiempo como por la intensidad alcanzada durante el conflicto.

Con respecto a esta última, las iniciativas para superar la confrontación en el país han sido múltiples, aunque los Acuerdos de la Habana han supuesto la culminación de este esfuerzo. Desde el año 2012, el gobierno colombiano, en cabeza del actual presidente, Juan Manual Santos Calderón, lideró un proceso de salida negociada a la confrontación armada con la insurgencia de las FARCEP. Como refrendo del Acuerdo de Paz alcanzado entre ambas partes se convocó un plebiscito que tuvo lugar día dos de octubre de 2016, resultando en el rechazo a la propuesta del acuerdo, una sorpresa para los pronósticos nacionales e internacionales.

Dichos resultados hacen necesario realizar estudios que permitan conocer los motivos que llevaron a tal rechazo al acuerdo de paz. Más concretamente, este artículo busca conocer la percepción que se tiene de la propuesta de paz entre el gobierno nacional y la insurgencia de las FARC-EP en la población del Tecnológico de Antioquia - Institución Universitaria, tratando de comprender la importancia de variables como el conocimiento del contenido de lo acordado o las fuentes de información a las que se recurre.

A través de la realización de encuestas a los docentes, alumnos y miembros del personal administrativo, y de su posterior análisis estadístico -a través del test de Fisher, de la relación policórica y de la regresión logística multinomial- se evidencian cómo existe una relación directa entre el conocimiento del contenido del acuerdo y la dirección del voto. Del mismo modo, se evidencia el importante papel que desarrollan los medios de comunicación en el proceso, no únicamente en la conformación de percepciones a favor o en contra del acuerdo, sino también en la difusión del contenido del mismo. 


\section{Contexto}

El artículo concluye analizando cómo de informada se encuentra la población del Tecnológico de Antioquia-IU con respecto al contenido del Acuerdo de Paz y cómo este influyó en la dirección del voto y en la fuente de conocimiento en la que el individuo se apoyaba. Del mismo modo, a nivel general se evidencian la diversidad de tendencias y percepciones que se establecen en torno al acuerdo de paz, y la potencialidad de la universidad pública en el proceso de difusión de los procesos internos que Colombia experimenta.

\section{Marco Teórico}

Teorías que soportan el tema de investigación

El concepto de percepción

Si bien la intención del presente artículo es realizar un estudio sobre la percepción del Acuerdo de Paz de la Habana, firmado entre el Gobierno Colombiano y las FARC-EP, y no es su objeto el estudio sobre acuerdos de paz, ni tampoco la psicología de la percepción; es válido primero realizar un acercamiento a estos términos que permitirán un mejor entendimiento del presente trabajo. Por ello, antes de avanzar en la revisión teórica acerca de plebiscitos, el voto en Colombia, etc., se hace imperativo definir qué es percepción, y qué es un acuerdo de paz.

Así, en cuanto al concepto de percepción existe una variada cantidad de teorías que abordan el tema desde distintos puntos, como la percepción social, ecológica, entre otras. De acuerdo a Kelly (1982) la percepción es un resultado compuesto por una sensación, originada por un estímulo; además de que la percepción consiste en experiencias presentes y pasadas. Por otra parte, acorde a la teoría de la Gestalt, la percepción se define como un "proceso de extracción y selección de información relevante, encargado de generar un estado de claridad y lucidez consiente que permita el desempeño dentro del mayor grado de racionalidad y coherencia posibles con el mundo circundante" (Oviedo, 2004, p. 90). Por último, desde la concepción 
El Acuerdo de Paz de la Habana. Un estudio...

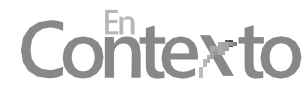
psicológica clásica, la percepción es un proceso activo-constructivo en el que un sujeto que percibe, antes de procesar la nueva información y con los datos archivados en su conciencia, se crea una visión que le permite anticipar, y que luego le lleva a contrastar el estímulo y aceptarlo o rechazarlo (Neisser, 1976).

Esta conceptualización de percepción es primordial dentro del análisis que se realiza en esta investigación ya que, en gran medida, parte del temor que se tiene por los Acuerdos de Paz de la Habana, se debe a la experiencia con otros procesos que ha tenido la sociedad colombiana en cuanto a la consecución de la paz con las antiguas FARC-EP. Tal es el caso de la negociación que se intentó entre el gobierno del entonces presidente Andrés Pastrana y dicha guerrilla en 1999. En este sentido, la percepción de la comunidad del Tecnológico de Antioquia puede estar ligada a la experiencia del aprendizaje, que en 1999 fue negativa.

Por otra parte, acuerdo de paz se entiende como un esfuerzo para lograr un convenio que ponga fin a la violencia, así como para implementarlo a través de negociaciones que pueden requerir la intervención de terceros (Fisas, 2010). $\mathrm{Y}$, adicionalmente, un proceso de paz es un concepto que se define por la actuación de actores de un conflicto que tratan de encontrar una salida al mismo, abarcando una pluralidad de elementos, entre las que se encuentra la negociación e implementación de los acuerdos (Darby, 2001).

\section{Referéndums y plebiscitos}

Desde hace décadas el recurso a referéndums y plebiscitos se ha incrementado notablemente, al entenderse que ofrecían soluciones a algunas de las principales carencias de representación de los sistemas democrático-liberales (Hobolt, 2009; Scarrow, 2001). En este sentido en los últimos dos años han tenido lugar, entre otros, el referéndum de permanencia en la Unión Europea por parte de Reino Unido, en Venezuela convocado por la Asamblea General en contra de las políticas del Presidente y en último lugar en Colombia para refrendar el acuerdo negociado entre el gobierno colombiano y las FARC-EP.

A través de la llamada directa a la ciudadanía se ha pretendido consolidar la legitimación de los gobiernos, así como la participación de los individuos en 
las decisiones políticas nacionales (Fishkin, 1995; Dyck \& Lascher, 2009). De hecho, este tipo de mecanismos es incluso percibido como un método para revertir los procesos contemporáneos de apatía política y dar solución a problemas propios de la globalización (Budge, 1996). En este sentido, existe una tendencia de aceptación de la población hacia los procesos de democracia directa. Tal comportamiento puede explicarse en una pluralidad de motivos, destacando por responder a las demandas de democracia directa o por mostrar una percepción de estabilidad (LeDuc, 2003).

No obstante, este apoyo no puede considerarse como homogéneo entre toda la población, ya que existen sectores que podrán verse más beneficiados. Más concretamente existen dos posturas que explican tal situación: la teoría de la movilización cognitiva y la teoría de la desafección política. La primera postura argumenta que los referéndums o plebiscitos benefician a aquellos sectores con mayores recursos cognitivos, ya que son quienes más van a percibir los beneficios de movilizarse políticamente en ellos (Inglehart, 1990). Por el contrario, aquellos con menores recursos más probablemente desconocerán las implicaciones de la consulta para sus intereses, mostrando por ello baja tendencia a la movilización.

Con respecto a la segunda teoría, esta defiende que los referéndums o plebiscitos suponen mecanismos de expresión para aquellos individuos que se encuentran desafectos o marginados con el sistema político, lo que implicará que sean dichos sectores los que más efectivamente se movilicen para participar en ellos (Norris, 1999). Según Schuck y De Vreese (2011, p. 197): "These citizens might hold critical views towards the political process but at the same time they stay committed to democratic principles and embrace the opportunity to have a say". Por el contrario, aquellos colectivos que ya se encuentren integrados en el sistema político nacional, tenderán a movilizarse menos por procedimientos alternativos como son los plebiscitos o referéndums. 


\section{Abstencionismo electoral en Colombia}

Si bien el abstencionismo es un problema a nivel internacional, Colombia destaca dentro del Continente Americano por ser uno de los países con más altos índices entre su población (Barrero, Liendo, Mejía \& Orjuela, 2013, p. 11). En el plebiscito sobre los Acuerdos de Paz de la Habana del día dos de octubre de 2016 tan solo un 37,43\% de la población llamada a votar ejerció su derecho al voto, cuestión que probablemente influyese en el resultado final de la consulta.

En este sentido, la abstención electoral puede tomarse desde dos puntos de vista: como el individuo que se cohíbe voluntariamente de ejercer su derecho al voto o como el fenómeno social que afecta al desarrollo del sistema político democrático (Torres, 2013, p. 8). Desde esta segunda perspectiva, el abstencionismo electoral se ha explicado principalmente desde tres factores: sociodemográficos, psicológicos y políticos. De esta manera se posibilita clasificar las razones por las cuales se genera el abstencionismo dependiendo de cada contexto temporal y material, además de abrir el debate normativo sobre las problemáticas derivadas del mismo y los mecanismos para resolverla (Alcubilla, 2017, p. 3).

Centrados en el caso de Colombia, desde una parte de la sociedad y de la academia se ha tomado la abstención como un efecto del pasado violento del país, que originó que las personas no votasen, al haber perdido la confianza en el gobierno y temer ser fruto de la violencia. A este suceso se unieron elementos infraestructurales del plebiscito - por ejemplo la falta de urnas en muchos sectores del país-, así como la propia crisis del sistema de partidos políticos (Barrero, 2013).

Adicionalmente, debe destacarse dos problemáticas, siendo la primera de ellas el fuerte desconocimiento del contenido del acuerdo entre la población colombiana. Por ejemplo, para el año 2015, el 65\% de los empresarios no conocían los acuerdos que se habían realizado en La Habana (El Tiempo, 2015). Con respecto a la segunda, se trata de la realización del acuerdo de paz con un secretismo en la negociación tanto hacia el conjunto de la población -al ser sus reuniones secretas- como hacia los gobiernos regionales y locales (Segura y Mechoulan, 2017) 
Medios de comunicación, conocimiento y voto.

A pesar de la discusión teórica sobre qué colectivos se movilizan más ante referéndums y plebiscitos, sí existe consenso acerca de la importancia que han venido representando los medios de comunicación en la forma en la que los ciudadanos perciben tales procesos de democracia directa. Así, dependiendo del tipo de aproximación se genera no sólo una mayor o menor preocupación, sino también la distinta percepción de la naturaleza del mismo -como una amenaza, como una oportunidad política, como un mecanismo de accountability, etc. (Raghubir and Menon, 2001).

En las últimas décadas, el gobierno colombiano ha tenido varios acercamientos hacia las guerrillas más fuertes dentro del país -FARC-EP y ELN principalmente-, teniendo los medios de comunicación un papel relevante en la difusión de tales procesos. No obstante, se ha considerado la existencia de un cierto sesgo en el cubrimiento de la información, tendiendo a mostrarse críticos hacia estas negociaciones (Bonilla Gomez, Borja, Iguarán Pimienta, \& López Ángel , 2008, pp. 48-49). Esto quiere decir que la manera en que los diferentes medios de comunicación presenten la información es también un desafío importante para el logro de la paz (Arteaga, 2012).

En este sentido, las críticas de los medios de comunicación se centraron en las penas a los combatientes de las FARC, rechazando que los guerrilleros no reciban penas de cárcel -sino penas privativas de libertad-y cuenten con cuotas de participación en el Congreso de la República de Colombia. Del mismo modo, fueron duramente criticadas las medidas de reinserción de los antiguos guerrilleros, considerándose los fondos públicos destinados como excesivos e inapropiados (Restrepo, 2016). Este sesgo, unido al secretismo con el que se desarrollaron las negociaciones, generaron un silencio gubernamental en el que las informaciones parciales o interesadas pudieron extenderse rápidamente entre la población nacional ${ }^{1}$.

\footnotetext{
${ }^{1}$ Cabe destacar también que en la campaña electoral por el plebiscito se vio un claro enfrentamiento entre Uribe y Santos -a su vez representantes de sectores políticos y económicos distintos.
} 
presidente impidieron un verdadero debate sobre el contenido del Acuerdo de Paz, lo que puede explicar que la población colombiana no se movilizara a las urnas y se alcanzase una abstención de más del 60 por ciento (Barrero, Liendo, Mejía \& Orjuela, 2013).

\section{Revisión de literatura}

La situación que se vivió en Colombia a causa de los inicios de los diálogos de paz en 2012 y su finalización en el año 2016, trajeron consigo grandes debates al interior del país que provocaron diversas confrontaciones que desembocaron en la lucha entre partidos políticos, así como un escenario de continuas tensiones. La situación por la que pasaba el país no fue solo del interés de Colombia ya que el mundo entero estaba a la espera de noticias sobre cuál sería su desenlace. Estos eventos, y todos los que se presentaron luego del tratado de paz, han generado en muchos lugares, tanto en Colombia como en otros países, interés en conocer el sentir del pueblo colombiano y en general las percepciones del mismo frente al proceso.

Una de las entidades interesadas en conocer estas percepciones fue la Universidad de Costa Rica, misma que en el 2015, realizó un estudio de percepción sobre los diálogos de paz que se desarrollaban en aquel tiempo a 500 jóvenes universitarios de la ciudad de Bogotá. El estudio fue desarrollado mediante encuestas, las cuales arrojaron resultados significativos sobre el apoyo de los jóvenes universitarios al proceso.

Esta investigación contiene preguntas cerradas como: “¿Está usted de acuerdo con que el gobierno de Santos consulte a la sociedad colombiana el contenido de un acuerdo con la guerrilla de las FARC? " de la cual se obtuvo un resultado significativo pues la mayoría de los encuestados, un 81,2\% (406 personas) dijeron que Sí y solamente el 12,4\% (62 personas) dijeron que No, lo que comprueba el gran apoyo que dieron los jóvenes al proceso de paz (Wilches \& Hernández, 2016, p. 42).

Entre las respuestas de las preguntas abiertas y de categorización variable se obtuvieron, en su mayoría, resultados favorables para el tratado de paz, como se puede evidenciar en los resultados obtenidos de las preguntas: "Establezca 
de 1 a 10, siendo 1 (mayor incidencia) y 10 (menor incidencia), el nivel de incidencia de los siguientes personajes en caso de darse un acuerdo de paz entre el gobierno Santos y la guerrilla de las FARC" en donde personajes como Juan Manuel Santos (presidente de Colombia) obtuvo 221 votos en un nivel de incidencia de 1, o un contraste particular en donde Álvaro Uribe Vélez obtuvo 32 votos con una incidencia de 1 (Wilches \& Hernández, 2016, p. 43).

Los resultados obtenidos de la pregunta abierta ¿Cuál sería el mecanismo idóneo para consultar el contenido de los acuerdos a la sociedad colombiana? demostraron que 212 personas consideraron que la consulta popular sería lo más correcto, 35 personas el plebiscito, 63 personas el referendo, 45 asamblea constituyente y 62 personas optaron por crear un nuevo mecanismo (Wilches \& Hernández 2016, p. 44).

Adicionalmente, un estudio realizado por Velez (2016) con base en información obtenida de LAPOP, arrojó que los jóvenes entre 18 y 25 años tenían más aceptación a la gran mayoría de preguntas del acuerdo, que las personas mayores de 25 años. Los motivos pueden ser diversos, pero se podría justificar esto porque los mayores de 25 años pudieron vivir épocas más violentas que el otro grupo. Preguntas como en una escala del 1(nada) al 7(mucho) sobre el apoyo al proceso de paz (Velez, 2016) el grupo de jóvenes de 18 a 25 años mostraron un apoyo del 4.38 mientras que los mayores de 25 años un 4.33 .

Además de estudios enfocados a las percepciones de los jóvenes en Colombia en cuanto al proceso de paz, también se han desarrollado investigaciones en cuanto a las percepciones de los colombianos sobre el proceso de paz desde una perspectiva de género. Un trabajo de investigación desarrollado por la Universidad de los Andes (Colombia) demuestra que no hay diferencia de género en cuanto al apoyo general del proceso de paz, sin embargo, en algunos casos como en la desmovilización definitiva y la reconciliación con los miembros de las FARC, las mujeres se muestran más escépticas que los 
hombres (Angulo, Ortiz, \& Pantoja, 2014). La investigación demuestra que es necesario evaluar y ver desde diferentes puntos de vista las respuestas indicadas por los encuestados ya que dependiendo de las circunstancias se puede comprender mucho mejor el porqué de la decisión de voto.

\section{Materiales y métodos.}

El presente estudio es de carácter exploratorio transversal, ya que se centra en recabar información en un momento específico en el tiempo. Concretamente, se toma la población del Tecnológico de Antioquia y se la divide en dos estratos (estudiantes y profesores- personal administrativo), procediéndose a continuación a aplicar un cuestionario de trece preguntas con relación a su percepción del Acuerdo de Paz entre el gobierno colombiano y las FARC-EP. Tabla 1.

Composición de la población del TdeA

\begin{tabular}{|l|l|l|l|}
\hline & Estudiantes & $\begin{array}{l}\text { Profesores } \\
\text { personal } \\
\text { administrativo }\end{array}$ & Total \\
\hline Población & 8069 & 721 & \\
\hline Muestra & 367 & 251 & $\mathbf{6 1 8}$ \\
\hline
\end{tabular}

Oficina de Talento Humano, TdeA (2016)

Con respecto a la población de profesores y personal administrativo, si bien se realizó una muestra para los dos colectivos, esta misma se ponderó en función del número de cada uno de ellos, repartiendo las 251 encuestas de la siguiente manera: 


\section{Contexto .González, A., Paradela, M.,Pineda, H., Areiza, S., García,

Tabla 2.

Composición de la población de profesores y personal administrativo del TdeA

\begin{tabular}{|l|l|l|}
\hline & Profesores & $\begin{array}{l}\text { Personal } \\
\text { administrativo }\end{array}$ \\
\hline Población & 629 & 92 \\
\hline Muestra & 218 & 33 \\
\hline
\end{tabular}

Oficina de Talento Humano, TdeA (2016)

Para obtener estadísticas con un 95\% de confiabilidad, se aplican 618 encuestas repartidas de la siguiente forma: 367 a estudiantes y 251 a profesores y administrativos (218 al primer grupo y 33 al segundo). Los datos utilizados para sacar las muestras corresponden al año 2016 y la técnica de muestreo desarrolladas es la del estratificado aleatorio -dado que se persigue realizar un estudio diferenciado con respecto a la respuesta a la encuesta en dichos estratos.

Tras aplicar el cuestionario a la muestra poblacional (en total 618 personas), la información obtenida se analiza mediante estadística descriptiva dentro del programa Data Analysis and Statistical Software (Stata) para realizar un estudio en tres niveles: en primer lugar un análisis básico de datos, centrado en la obtención de porcentajes y tablas estadísticas; en un momento posterior se realiza la comparación entre los dos sectores sobre los que se ha realizado la muestra (estudiantes y profesores-administrativos), buscando las diferencias entre ellos; y en último lugar el estudio se centra en el análisis de las correlaciones entre las preguntas, especialmente centradas en la orientación del voto, el conocimiento del acuerdo y los medios de información. 
Para ello se recurren a diferentes mecanismos estadísticos, entre los que destaca la regresión logística multinomial, que permite detectar las diferencias entre los tres estratos; el test de Fisher, que posibilita comprobar las correlaciones existentes entre las diferentes variables estudiadas; y la correlación policórica, que indica el sentido de las correlaciones en variables categóricas.

\section{Análisis de resultados}

Tal y como se expuso anteriormente, los datos obtenidos se han analizado con el propósito de demostrar tendencias generales en el conjunto de la población del TdeA, así como a poner en evidencia las diferencias entre alumnos y docentes-administrativos.

En primer lugar, se ha estudiado la respuesta a la pregunta 1, referente al voto en el plebiscito sobre el Acuerdo de Paz. En este sentido puede observarse que la postura predominante ha sido el voto a favor del acuerdo, alcanzando un $35,9 \%$ de los encuestados, frente al 32,3\% que votó en contra del mismo. Esta diferencia de tres puntos, es muy relevante, al tener en cuenta que la diferencia entre el voto por el sí y por el no en el Departamento de Antioquia y de Medellín fue de 24 puntos en contra del Acuerdo de Paz.

Del mismo modo destaca el alto porcentaje de abstencionismo, que alcanzó a un $20,8 \%$ de los encuestados, incrementándose hasta casi un $25 \%$ si se suma el $4 \%$ de quienes sí votaron, pero lo hicieron en blanco o voto nulo. Evidencia, en todo caso que más de uno de cada cuatro encuestados del Tecnológico de Antioquia no ejerció su derecho al voto o no se sintió representado por ninguna de las posturas, un dato relevante a tener en cuenta. 


\section{Contexto .González, A., Paradela, M.,Pineda, H., Areiza, S., García,

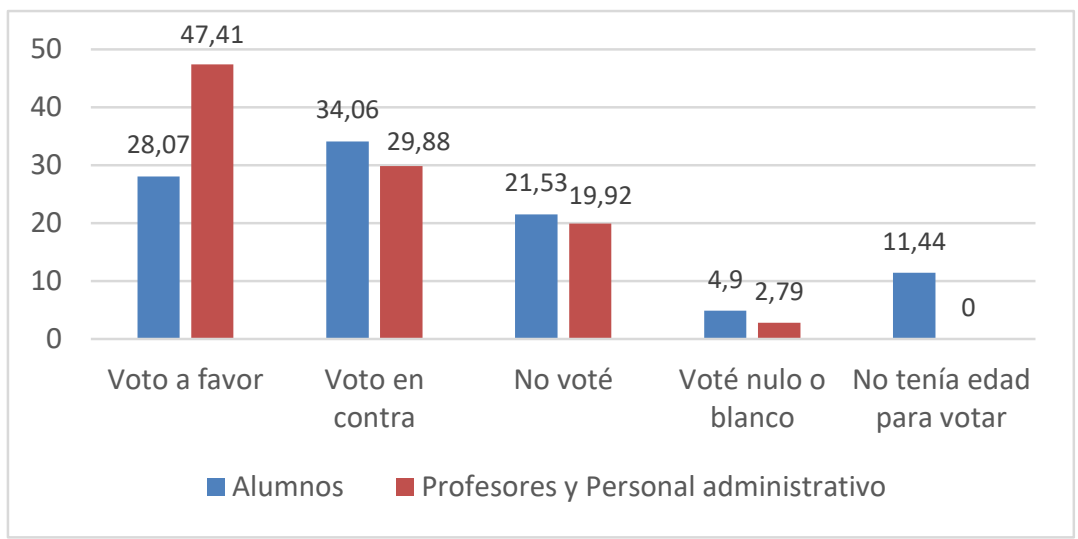

Figura 1. Voto en el plebiscito.

Elaboracion propia

No obstante, el análisis ha demostrado una importante diferencia intergrupal entre alumnos por un lado y profesores y personal administrativo por otro. En primer lugar, el porcentaje de profesores y administrativos que votaron a favor fue superior a los alumnos en casi veinte puntos. Por el contrario, el voto en contra refleja un resultado opuesto, puesto que los alumnos les superan en más de cinco puntos. Por ende, puede afirmarse que existe una diferencia sustancial en la orientación del voto y la situación educativa o laboral del encuestado.

Por otro lado, también es relevante analizar la pregunta 2, que plantea si se considera que el Acuerdo de Paz beneficia al conjunto de la sociedad colombiana. A este respecto un 55,5\% de los encuestados manifestaron que existe tal beneficio, frente al $44,5 \%$ que no lo cree. Estos resultados arrojan importante relevancia, dado que la diferencia de 11 puntos es casi 7,4 puntos mayor a la diferencia entre el voto a favor y en contra. Esto implica indudablemente que encuestados que no votaron, lo hicieron en blanco o nulo, o votaron en contra, al mismo tiempo consideran positivo el Acuerdo de Paz. 
Tabla 3.

Valoración del beneficio del Acuerdo de Paz

\begin{tabular}{|l|}
\hline 0. Considera que no \\
beneficia a la sociedad \\
colombiana. \\
1. Considera que \\
beneficia a la sociedad \\
colombiana. \\
\hline
\end{tabular}

. tab pregunta2
\begin{tabular}{r|rrr} 
pregunta2 & Freq. & Percent & Cum. \\
\hline 0 & 275 & 44.50 & 44.50 \\
1 & 343 & 55.50 & 100.00 \\
\hline Total & 618 & 100.00 &
\end{tabular}

Elaboración propia.

Siguiendo con el análisis, a continuación se ha creado una variable a partir de las respuestas a las preguntas $5,6,7$ y 8 , en las que se pregunta acerca del conocimiento de las cuatro partes principales del acuerdo: Reforma Rural Integral, Participación Política, Alto al Fuego y Desarme Permanente y Sistema Integral para las Víctimas del Conflicto. A partir del mismo se ha evaluado el nivel de conocimiento de los alumnos sobre el conjunto del Acuerdo de Paz, distinguiendo entre "Conocimiento completo" (sobre las 4 partes), "Conocimiento avanzado" (3 partes), "Conocimiento normal" (2 partes), "Conocimiento bajo" (1 parte) y "Conocimiento nulo" (0 partes).

Así, resulta relevante en primer lugar constatar que el 28,8\% de los encuestados conoce las cuatro partes del Acuerdo de Paz. Por el contrario, un $23 \%$ desconoce el contenido completo del acuerdo, alcanzando el 56\% aquellos que desconocen como mínimo dos de las cuatro partes del acuerdo. Esto evidencia sin duda que una parte de los votos emitidos en el Plebiscito no fue adecuadamente informada, lo que sin duda tuvo efectos importantes en el resultado del mismo. 


\section{Contexto .González, A., Paradela, M.,Pineda, H., Areiza, S., García, C., Loaiza, K. y Parra, J.}

Tabla 4.

Conocimiento del acuerdo (en frecuencia y porcentajes)

. tab conacuerdo
\begin{tabular}{r|rrr} 
conacuerdo & Freq. & Percent & Cum. \\
& & & \\
0 & 142 & 22.98 & 22.98 \\
1 & 81 & 13.11 & 36.08 \\
2 & 127 & 20.55 & 56.63 \\
3 & 90 & 14.56 & 71.20 \\
4 & 178 & 28.80 & 100.00 \\
\hline Total & 618 & 100.00 &
\end{tabular}

Elaboración propia.

Por otro lado, aplicando de nuevo un análisis intergrupal, puede diferenciarse una clara divergencia en función del sujeto encuestado. A nivel general puede observarse un mayor conocimiento del Acuerdo de Paz por parte del personal docente y administrativo, superando en más de veinte puntos el porcentaje que tiene un conocimiento completo del acuerdo. Sin embargo, también es reseñable que superan en más de tres puntos y medio el porcentaje que tiene conocimiento nulo del acuerdo. Por tanto, si bien es cierto que existe una mayor tendencia a conocer el acuerdo en el colectivo de profesores y personal administrativo, esta afirmación no puede definirse como plenamente causal. 


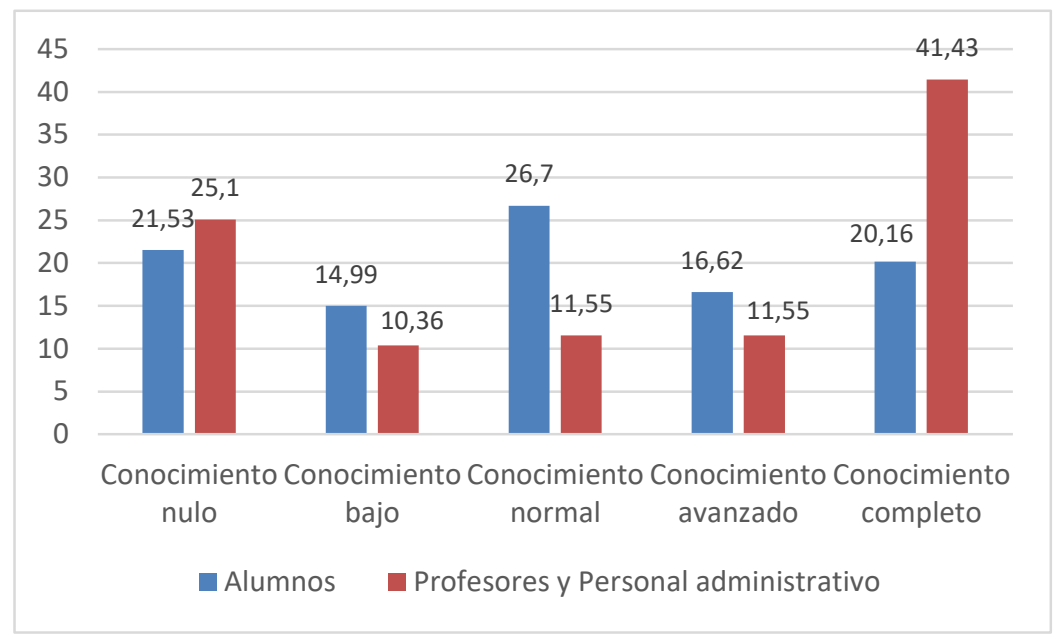

Figura 2. Conocimiento del acuerdo con diferenciación intergrupal Elaboración propia.

Por otro lado, debe estudiarse si existe una correlación directa entre el nivel de conocimiento y la dirección del voto. Para ello se realiza el test de Fisher a la correlación entre ambas preguntas, agrupando el voto en tres sectores ("Votó a favor", "Votó en contra" o "Otras opciones"). Del mismo modo, por motivos organizacionales, se ha agrupado la variable de conocimiento en tres sectores: Bajo (incluye nulo y bajo), Medio (incluye normal) y Alto (incluye avanzado y completo). El resultado de 0,00 en el mismo, evidencia que existe una relación estadísticamente significativa entre ambas variables ${ }^{2}$ : el conocimiento que el alumno tiene del Acuerdo de Paz influyó claramente en su decisión de voto.

\footnotetext{
${ }^{2}$ Para que pueda afirmarse que existe una correlación debe obtenerse un resultado por debajo de 0,005 .
} 


\section{Contexto .González, A., Paradela, M.,Pineda, H., Areiza, S., García,

Tabla 5.

Aplicación del Test Fisher a la correlación entre voto conocimiento deAcuerdo de Paz

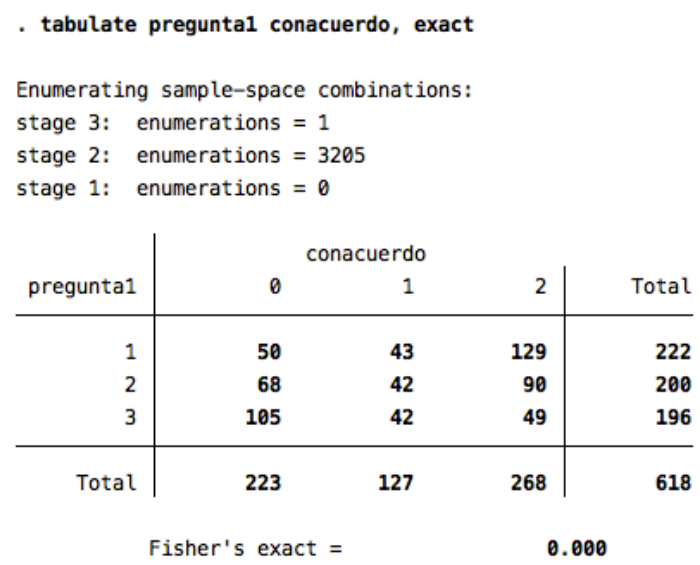

Elaboración propia

Continuando con el análisis, el resultado de computar la correlación policórica muestra un coeficiente de -0.37 , lo cual indica que las personas que votaron a favor del Acuerdo de Paz tienen una tendencia a conocer el Acuerdo de Paz un $63 \%$ más que las otras opciones. Evidencia, en definitiva, que quienes votaron a favor del acuerdo tenían un mayor conocimiento del contenido del mismo, lo que abre la puerta a otra serie de debates.

Tabla 6.

Aplicación de la correlación policórica entre conocimiento del acuerdo y dirección del voto

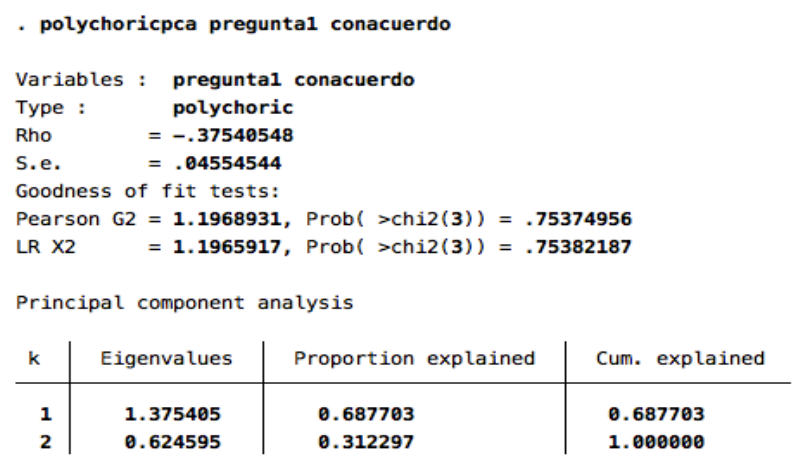

Elaboración propia. 
Por otro lado, se analizó si existe una relación entre la orientación del voto y el haber sido directa o indirectamente objeto de la violencia del conflicto, cuestión abordada en la pregunta 9. No obstante, la aplicación del test de Fisher con un resultado de 0,023 evidencia que la posibilidad de que no exista correlación entre ambas variables no es descartable. Por tanto, no puede afirmarse que para los estudiantes, docentes y personal administrativo del Tecnológico de Antioquia, el haber sido afectado por el conflicto, directa o indirectamente, influya en la determinación de su voto con respecto al Acuerdo de Paz.

Por último, se ha realizado un estudio de los medios de información sobre los que los encuestados se han basado, arrojando un primer resultado ciertamente relevante: únicamente el $1,8 \%$ de los encuestados afirman no haberse informado regularmente sobre el Acuerdo de Paz. Entre el resto, los medios de información con más impacto son la televisión y los periódicos, siendo que el $76,9 \%$ y el $57,9 \%$ de los encuestados se informaron regularmente a través de ellos.

Resulta destacable este resultado si se pone en consonancia con las preguntas $5,6,7$ y 8 resumidas en la figura número dos, que evidencia un conocimiento en promedio bajo sobre el contenido del Acuerdo de Paz. Sucede, en definitiva, que la población encuestada afirma informarse de manera regular y variada sobre el Acuerdo de Paz pero al mismo tiempo mayoritariamente desconoce su contenido. Tal paradoja parece apuntar a que la labor informadora de los medios de comunicación fracasó en el proceso del plebiscito y que, de hecho, lo ha seguido haciendo durante el año de implantación del nuevo acuerdo. 


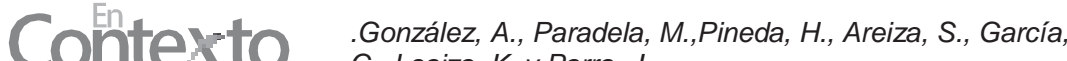

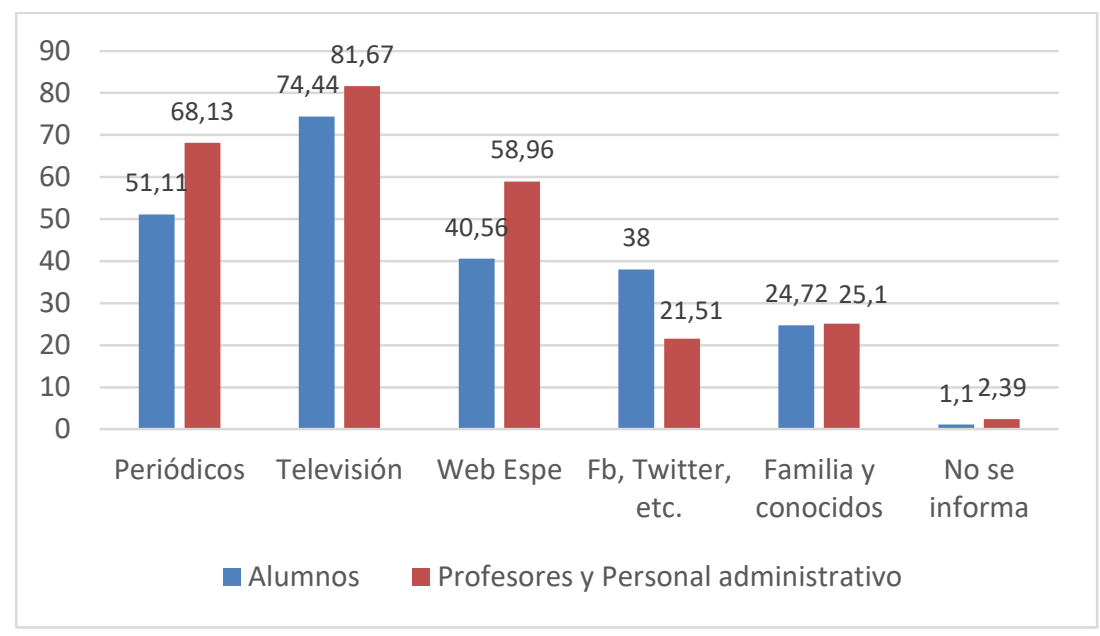

Figura 3.Distribución de los medios de información de la población del Tdea. Elaboración propia

Adicionalmente, el estudio de las diferencias entre los dos grupos muestra resultados relevantes: si bien en general existen importantes similitudes, profesores y administrativos se informan un $28 \%$ más en medios de información especializados y un $17 \%$ menos en medios no especializados. Con el propósito de demostrar si existía una relación directa entre estos hechos se realizó el test de Fisher, demostrando una clara tendencia entre el voto de los encuestados y su información en medios de comunicación especializados. Complementado con la correlación policórica, puede afirmarse que los que se informaron a través de medios de comunicación especializados tuvieron un $67 \%$ más de posibilidades de votar a favor del acuerdo que los que no lo hicieron. 
Tabla 7.

Aplicación de la correlación policórica entre la utilización de medios de comunicación especializados y dirección del voto

. polychoricpca preguntal pregunta10pagesp

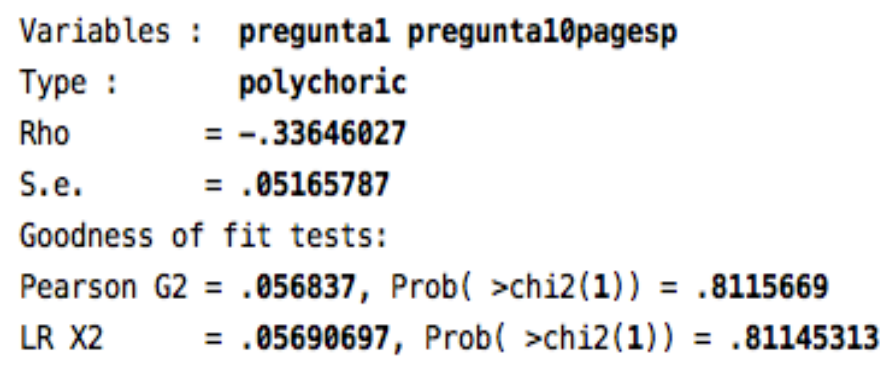

Principal component analysis

\begin{tabular}{l|c|c|c}
$\mathrm{k}$ & Eigenvalues & Proportion explained & Cum. explained \\
\hline 1 & 1.336460 & 0.668230 & 0.668230 \\
2 & 0.663540 & 0.331770 & 1.000000
\end{tabular}

Elaboración propia.

Por último, se realiza una regresión logística multinomial, en la que se comprueba que efectivamente existe una relación entre el conocimiento del acuerdo y la dirección del voto. El resultado señala que la probabilidad de votar en contra del acuerdo decrece en una intensidad de 0.65 cuanto más alto es el conocimiento del contenido del acuerdo. En tal proceso se han tomado en consideración otra serie de variables (preguntas 2, 3 y 9) para medir el nivel de interferencia en la relación entre ambas variables. Con ello se ha tratado de minimizar las posibles fuentes de endogeneidad. 


\section{Contexto .González, A., Paradela, M.,Pineda, H., Areiza, S., García,

Tabla 8 .

Regresión logística multinomial aplicada a la relación entre el conocimiento del Acuerdo de Paz y la dirección del voto

\begin{tabular}{c|rlrrrr}
\hline & \multicolumn{7}{c}{} & & & & & \\
conacuerdo & & & & & & \\
1 & -.8300417 & .3137468 & -2.65 & 0.008 & -1.444974 & -.2151093 \\
2 & -1.654935 & .2741759 & -6.04 & 0.000 & -2.19231 & -1.11756 \\
& & & & & & \\
ocupacin & -.7843165 & .2443914 & -3.21 & 0.001 & -1.263315 & -.3053182 \\
pregunta9 & -.3411126 & .1612449 & -2.12 & 0.034 & -.6571468 & -.0250783 \\
pregunta2 & -2.367201 & .3471924 & -6.82 & 0.000 & -3.047685 & -1.686716 \\
pregunta3 & .050582 & .1924371 & 0.26 & 0.793 & -.3265878 & .4277518 \\
pregunta4 & -.6324565 & .2751652 & -2.30 & 0.022 & -1.17177 & -.0931426 \\
_cons & 4.186415 & .6526896 & 6.41 & 0.000 & 2.907167 & 5.465663 \\
\hline
\end{tabular}

Elaboración propia.

En definitiva, el análisis estadístico de las encuestas realizadas arroja una relación causal entre el nivel de conocimiento del Acuerdo de Paz, los medios de información utilizados por el encuestado para conocer el acuerdo y la dirección final del voto.

\section{Conclusiones.}

Dado el análisis realizado en el punto anterior, en función de los datos empíricos pueden argumentarse que existe una diferenciación inter-grupal más que relevante, es decir, que existen diferencias significativas entre alumnos y docentes- administrativos en cuanto a la percepción del Acuerdo de Paz. Más concretamente, se han demostrado diferencias sustanciales en la orientación de voto (el alumnado votó en mayor porcentaje en contra del acuerdo), en el conocimiento del contenido del acuerdo (profesores y administrativos conocen significativamente mejor el Acuerdo de Paz) y en los mecanismos de información (los alumnos acudieron en mayor porcentaje a medios no especializados que los profesores y administrativos).

Adicionalmente, del análisis de los datos globales de las encuestas se sustrajeron una serie de conclusiones, destacando dos de ellas: que parte de los encuestados que no votaron a favor del acuerdo sí consideraban que era 
El Acuerdo de Paz de la Habana. Un estudio...

beneficioso para la sociedad colombiana y que un porcentaje significativo de los mismos afirmaron informarse habitualmente a través de los medios de comunicación sobre el Acuerdo de Paz, a pesar de tener un conocimiento bajo o muy bajo del contenido del acuerdo.

Del mismo modo, el estudio a través del test de Fisher y de la correlación policórica evidenció una serie de relaciones causales dentro de las respuestas a las encuestas. Más concretamente, se probó que había una relación entre el bajo conocimiento del contenido del Acuerdo de Paz y el voto en contra del mismo, así como en la utilización de medios de información especializados y la tendencia a votar a favor en el Plebiscito. Por otro lado, resulta relevante el no haber encontrado resultados significativos en la relación entre el voto emitido y la violencia sufrida durante el conflicto con las FARC-EP: según lo evidenciado por este artículo, el haber sido o no objeto de la violencia no influyó en el sentido del voto sobre el Acuerdo de Paz.

Por otro lado, ha sido relevante el análisis intergrupal, que ha mostrado diferencias significativas entre los dos grupos estudiados. Así, docentes y administrativos han tendido a votar en mayor porcentaje a favor del Acuerdo de Paz, a tener un conocimiento superior del contenido del mismo y a informarse en mayor medida por medio de información especializada. Por el contrario, el alumnado ha votado mayoritariamente en contra del acuerdo, mostró un nivel de conocimiento del acuerdo en promedio más bajo y se informó en menor medida en fuentes especializadas.

Dispuestas estas evidencias estadísticas, pueden realizarse una serie de conclusiones a través de su interpretación. En primer lugar, con respecto a las fuentes de información, puede afirmarse que tienen una gran importancia a la hora de informar al votante e incluso de determinar su voto. Así mismo, puede sostenerse que el nivel de desconocimiento de los diferentes grupos también guarda una relación muy estrecha con la decisión del voto, confirmando así la relevancia que tiene no sólo en la participación ciudadana, sino también en su nivel de compromiso con buscar fuentes de información confiables. De hecho, la regresión logística multinomial consolidó estadísticamente los datos anteriores, evidenciando que existía una relación entre el hecho de tener un mayor conocimiento del contenido del Acuerdo de Paz y el voto en apoyo del 


\section{Contexto .González, A., Paradela, M.,Pineda, H., Areiza, S., García, \\ mismo.

Queda reafirmado, por ende, la importancia de que exista una transmisión de conocimiento por parte de la universidad pública, tanto desde una perspectiva general, con actos de información, como a través de unos profesores formados y conocedores de los procesos sociales que Colombia experimenta. Se plantea como un futuro análisis por parte de este semillero la realización de un estudio cualitativo que incida en las causas de las diferencias intergrupales y que permia ahondar en los resultados obtenidos en este estudio.

\section{Referencias}

Alcubilla, E. (2017). Abstencionismo electoral. Recuperado de http://www.corteidh.or.cr/tablas/14910.pdf

Angulo, M. C., Ortiz, A. M., \& Pantoja, S. D. (2014). Análisis de las percepciones de los colombianos sobre el proceso de paz y el posconflicto desde una perspectiva de género. Colombia Internacional, 80. Recuperado el 27 de Septiembre de 2017, recueprado de: http://www.scielo.org.co/scielo.php?script=sci_arttext\&pid=S0121 56122014000100007

Arteaga, B., Walteros, D., Álvarez, L., Andrade, O., Puentes, J., Cortés, A., y Baños, L.C. (2012). Cuadernos Paz a la Carta número ${ }^{\circ}$. Recuperado el 27 de Agosto de 2017. Recuperado de: http://www.utadeo.edu.co/files/collections/documents/field_attached_ file/cuaderno2.pdf

Barrero, F., Liendo, N., Mejía, L., \& Orjuela, G. (2013). Abstención electoral en Colombia: una apróximación a sus causas. Bogotá. Recuperado el 26 de Agosto de 2017. Recuperado de http://www.registraduria.gov.co/IMG/pdf/CEDAE_-

_Abstencionismo_electoral_en_Colombia.pdf 
Bonilla Gomez, J., Borja, P., Iguarán Pimienta, J., \& López Ángel , M. C. (2008). Lo que construimos desde abajo: comunicación para la paz en Colombia. (Tesis de pregrado). Universidad Javeriana, Bogotá. Recuperado de: http://www.javeriana.edu.co/biblos/tesis/comunicacion/tesis113.pdf Budge, I. (1996). The New Challenge of Direct Democracy. Cambridge, Reino Unido: Polity Press.

Barrero, F. (2013). Abstencionismo electoral en Colombia: una aproximación a sus causas. Bogotá, Colombia: Digiprint.

Darby, J. (2001). The Effects of Violence on Peace Processes. Washington, EEUU: United States Institute of Peace.

Dyck, J.; Lascher, E. (2009). Direct Democracy and Political Efficacy Reconsidered. Political Behavior, 31, 401-427.

El Tiempo. (2015, 19 de Agosto). El $65 \%$ de los empresarios no conoce acuerdos logrados con las Farc. El tiempo. http://www.eltiempo.com/archivo/documento/CMS-16255455

Fisas, V. (2010). Anuario Procesos de Paz. Escola de Cultura de Pau. Barcelona, España: Icaria Editorial.

Fishkin, J. (1995). The Voice of the People: Public Opinion and Democracy. New Haven, EEUU: Yale University Press.

Hobolt, S. (2009). Europe in Question: Referendums on European Integration. Oxford, Reino Unido: Oxford University Press.

Inglehart, R. (1990). Culture Shift in Advanced Industrial Society. Princeton, EEUU: Princeton University Press.

Kelly, W.A. (1982). Psicología de la Educación. Psicopedagogía fundamental $y$ didáctica. Aplicaciones especiales y eneseñanza programada. Madrid, España: Ediciones Morata. 


\section{Contexto .González, A., Paradela, M.,Pineda, H., Areiza, S., García,}

C., Loaiza, K. y Parra, J.

LeDuc, L. (2003). The Politics of Direct Democracy: Referendums in Global Perspective. Peterborough. Toronto, Canadá: University of Toronto Press.

Neisser, U. (1976). Cognition and reality: Principles and implications of cognitive psychology. New York, EEUU: Henry Holt \& Co.

Norris, P. (ed). (1999). Critical Citizens: Global Support for Democratic Governance. Oxford, Reino Unido: Oxford University Press.

Oviedo, G.L. (2004). La definición del concepto de percepción en psicología con base en la teoría Gestalt. Revista de Estudios Sociales, 18, 89-96.

Raghubir, P.; Menon, G. (2001). Framing Effects in Risk Perception of AIDS. Marketing Letters, 12, 145-155.

Restrepo. J. (2016). Colombia: nota sobre los borradores de La Habana hasta ahora. En Alvarado. S; Rueda, E.; Gentili, P. (Ed.), Paz en Colombia: perspectivas, desafíos y opciones. Buenos Aires, Argentina: CLACSO.

Segura, R.; Mechoulan, D. (2017). Made in La Havana: cómo Colombia y las FARC decidieron terminar la guerra. Nueva York, EEUU: International Peace Institute.

Scarrow, S.E. (2001). Direct Democracy and Institutional Change: A Comparative Investigation. Comparative Political Studies, 34, 651665.

Schuck, A.; De Vreese, C.H. (2011). Public Support for Referendums: The Role of the Media. West European Politics, 34, 181-207.

Torres, A. (2013). Abstención electoral en Colombia. Desafección política, violencia política y conflicto armado. Recuperado de http://politicasysociologia.ucm.es/data/cont/docs/21-2016-12-21CI12_W_Ana\%20Patricia\%20Torres.pdf

Velez, G. (2016). ¿Y qué piensan ellos? Jóvenes colombianos y sus actitudes sobre la paz. La silla vacia. http://lasillavacia.com/silla-llena/red-dela-paz/historia/y-que-piensan-ellos-jovenes-colombianos-y-susactitudes-sobre-la.

Wilches, J. A.; Hernández, M. (2016). Jóvenes universitarios: percepciones y encuestas sobre conflicto armado y paz en Colombia. Reflexiones 95, 33-55. 
El Acuerdo de Paz de la Habana. Un estudio...

Contexto 